\section{Fuzzy Systems Evaluation: The Inference Error Approach}

\author{
A. Sala, P. Albertos
}

Abstract - An inference method based on an equation image of a rulebase and the minimisation of a cost index (inference error) is here presented. The method has several advantages: intuitive insight, allows more powerful rules, and also presents an interesting algebraic (minimum distance) and functional (subsethood) formulation of the meaning of inference. Analysis of internal quality and completeness of a fuzzy rulebase and validity checks of inference methods can be expressed as numeric indexes. This is applied to logical verification of common structures widely used in PID fuzzy controllers.

\section{INTRODUCTION}

Fuzzy logic [12] is a key element in the application of rulebased systems to engineering (process control, diagnosis, etc.) by integration of human knowledge. Nevertheless, there are various implication [4] and defuzzification methods [6], most of them intuitively clear but having 'fat shape' problems or erroneus output with nonconvex sets. To avoid this, other algorithms rely on entropy coefficients or heuristics[11].

In industry applications [1], the choice of fuzzy operators, defuzzification methods and rulebase construction, are based on easiness of implementation, user understandability and experience, without any further optimisation or validation. Many current practical fuzzy systems carry out mere interpolation under a reasoning mask, alike to human solutions to some problems but unnecesarily restricting fuzzy logic possibilities.

In the field of expert system verification and validation [7], there is a need to generalise two-valued logic verification to the fuzzy case. Automated binary logic tools [8] based on graphs, decision tables [3], Petri nets, etc. have been devised. In this paper, validation results are based on a generalisation of contradiction from binary logic, being expressed as a cost index (inference error) to be minimised.

The methods here presented define an ideal inference as a reference point for comparison of other algorithms and rulebase logic validation: for a coherent rulebase, inference will be analogous to solving a set of equations; the concept will be generalised so the correct conclusion will be the one that minimises contradiction. This will allow extracting conclusions even from inconsistent rulebases. An interpretation based on the extension principle [13] presents rules as examples of the function a rulebase models. This provides interesting insights into fuzzy control design. See [5] for an alternate interpretation from vague environments and extensional mappings.

Apart from providing validation methods of fuzzy inference algorithms, the equation approach will provide ways to differentiate IF from IIF rules and allow conclusions as ' $x$ is $A$ OR $y$ is $B$ ', that do not easily fit in conventional paradigms.

The paper is structured as follows: first, rule equations are presented. Contradiction and completeness are defined as existence and uniqueness of solutions. Third section presents rulebases as function approximators, formalising some heuristics.

Manubscript received ....

The authors are with Universidad Politécnica de Valencia, Aptdo. 22012, E-46071 VALENCIA (SPAIN), E-Mail : asala@ aii.upv.es
The formulation of the inference error contradiction measure is presented in section 4. The validation of centroid defuzzifiers using triangular memberships in look-up table fuzzy controllers is reported in the fifth section. A conclusion section will stress the most important results.

\section{INFERENCE, RULES AND EQUIVALENT EQUATIONS}

Logic, knowledge. In logic-based systems, knowledge is usually available in both implication form (rules) and atomic propositions with an associated truth value (premises). Twovalued logic deals with operators defined over the $\{0,1\}$ ( $\{$ false, true $\})$ set. In fuzzy logic, the truth values lie in the interval $[0,1]$. Conjunction, disjunction and negation are triangular norms, conorms and 1-complement, respectively. IMPLICATION $(\Rightarrow)$ and DOUBLE IMPLICATION $(\Leftrightarrow)$ operators representing rules are analysed in this paper. Generalisation of binary logic forms, such as $-A+B$, is not obvious, leading to various interpretations [4].

Sets, concepts. Given a universe set $U$ and a set $A \subseteq U$, " $x$ is A"-true is defined equivalent to $x \in A$. Concepts admitting quantification of meaning can be represented by fuzzy sets [12], defined by membership functions $(\boldsymbol{M F})$ : the grade of membership to a fuzzy set $F \subseteq U$ is expressed by a function $\mu_{\mathrm{F}}: U \rightarrow[0,1]$. Fuzzy set intersection, union and complementation are generalised with the appropriate fuzzy logic operators over their MF's. Generalisation of subsethood to fuzzy sets is an inequality: given two fuzzy sets $A$ and $B, A \subseteq B$ if and only if $\mu_{A}(x) \leq \mu_{B}(x) \forall x \in U$.

Inference. Given some premises, additional knowledge can be inferred by proving truth values of a set of conclusions so that no contradiction arises. Well established techniques are used in the binary case. They cannot be carelessly extended to the fuzzy case.

\section{A. Fuzzy inference equivalent equations.}

An equation image of fuzzy rulebases may be formed by an implication equation that meets binary generalisation requirements. For various reasons, the Lukasiewicz bounded sum (" $\mathrm{A} \Rightarrow \mathrm{B}$ is true" equivalent to $\min (1,1-A+B)=1$ ) is advantageous over other choices, such as "Not A Or B is true" $\equiv \max (1-A, B)=1$. The former is equivalent to a simpler inequality, used as a basic definition for implication.

Definition 1. The equivalent inequality that replaces fuzzy implication " $A \Rightarrow B$ - true" is defined as: $\quad A \leq B$

Thus, the double implication " $A \Leftrightarrow B$ - true", represented as $A \leq B$ and $B \leq A$ will be equivalent to the equation: $A=B$

In this way, inference is transformed into solving a set of equalities and inequalities. In composite propositions using the AND, OR, and NOT logic operators, the algebraic representation will be: $\min (A, B), \max (A, B)$ and $1-A$.

A similar choice has been taken into consideration by some authors (see [9]). The Gödel-Brouwer implication and other $R$ implications [4] lead to the same inequality. The extension principle and its relation with subsethood will prove that this option has interesting properties (section 3).

Remark. Fuzziness is only existent in premises. Rules are assumed stated as " $\mathrm{A} \Rightarrow \mathrm{B}$ is TRUE". Rule truth in the interval $(0,1)$ may lead to presumption, prejudice and paradoxes [9]. Intermediate cases will be dealt with by confidence functions, as 
reported in section 4 .

If implication equations are combined with fuzzifiers (membership functions for sets $A, B$ over universes $U$ and $V$ for variables $x$ and $y$, respectively) they present the form:

$$
\mu_{A}(x) \leq \mu_{B}(y) \quad \mu_{A}(x)=\mu_{B}(y)
$$

for "IF $x$ is $A$ Then $y$ is $B$ " and its IIF version, respectively.

Definition 2. Given a fuzzified rule as in (3), the set of $y$ that are solution of (3) (given $x$ ) will be named conclusion set of the rule. A rule is fired if its conclusion set is not the whole universe.

Note. By using equations no defuzzification is needed in inference: ideally, only fuzzification exists. Defuzzifiers are just algorithms devised to actually carry out the equation solving (maybe an approximation of it) ; they are not a basic theoretical entity. If some rules are contradictory then conflicts arise and no solution exists (individual conclusion sets are disjoint). (See B.)

Other properties. Equation representation is a convenient way to derive properties coming from intuitive insights or from generalisations of binary logic, as pointed out by the following:

Proposition 1 (subsethood). If $U \equiv V$ in equation (3) then :

"IF $\mathrm{x}$ is $\mathrm{A} \rightarrow \mathrm{x}$ is $\mathrm{A}^{*} " \Leftrightarrow \mu_{A}(x) \leq \mu_{A^{*}}(x) \Leftrightarrow A \subseteq A^{*}$

Proposition 2. ' $A \Rightarrow(B \text { and } C)^{\prime}$ - $A \leq \min (B, C)$ - is equivalent to $\left\{{ }^{\prime} A \Rightarrow B\right.$ ', $\left.{ }^{\prime} A \Rightarrow C C^{\prime}\right\} \equiv\{A \leq B, A \leq C\}$. ' $(A$ or $B) \Rightarrow C$ '” $\equiv\left\{{ }^{\prime} A \Rightarrow C\right.$ ' ${ }^{\prime} B \Rightarrow C$ ' $\}$.

Proposition 3. (Chaining). As $A \leq B, B \leq C$ imply $A \leq C$, from $\{$ ' $A \Rightarrow B$ ' , ' $B \Rightarrow C$ ' $\}$ rule ' $A \Rightarrow C$ ' is derived.

Definition 3. Two sets $M, N$ defined over a certain universe are "fuzzy mutually exclusive" if $\forall x \mu_{M}(x)+\mu_{N}(x) \leq 1$.

The definition arises because the postulated equation is that of "If $x$ is $M$ then $x$ is Not $N$ ": membership sum of 1 is the maximum "overlapping" for mutual exclusion. This is very popular indeed in rulebase design (sect. 5), analogue to sets $M$ and $N$ being disjoint over the Lukasiewicz $t$-norm [5].

\section{B. Rulebase analysis and formal verification.}

The utility of the presented algebraic image of rules is clear when defining concepts such as coherence, contradiction and redundancy in terms of existence and uniqueness of solutions of the equation set formed by replacing each rule by its equation (3).

Definition 4. If, for a given set of premises, there exists at least one solution to the equation set, the rulebase is said to be coherent for these premises.

Definition 5. If, for a given set of premises, there exists exactly one solution, the rulebase is said to be complete for the premises.

Definition 6. If, for a given set of premises, there exists no solution, the rulebase is said to be contradictory for the premises.

Definition 7. If the elimination of an equation does not change the solutions, the rule as well as the rulebase are said to be redundant.

Definition 8. A candidate conclusion is said to be coherent with a rulebase and its premises if it is a solution of the related equation set (maybe it is not the only one).

A rulebase is coherent if the intersection of all of the conclusion sets (Def. 2) is not empty. The inference error (section 4) evaluates a distance between conclusion sets in case of conflict, to distinguish "absolutely contradictory" from "nearly coherent" rulebases.

Example. The following fuzzy rulebase :

1-If $x$ is Low Then $y$ is Not Low and $z$ is Low

2-Only if $x$ is Medium Then $y$ is Low
3-If $x$ is High Then $y$ is High or $z$ is High

is defined equivalent to the set of equations:

$$
\begin{aligned}
& \operatorname{Low}(x) \leq \min (1-\operatorname{Low}(y), \operatorname{Low}(z)) \\
& \operatorname{Medium}(x)=\operatorname{Low}(y) \\
& \operatorname{High}(x) \leq \max (\operatorname{High}(y), \operatorname{High}(z))
\end{aligned}
$$

Given a particular $x$, a solution to the equations (values of $y$ and $z$ verifying them) is, by definition, a conclusion of the inference. Rule 1 is equivalent to \{ If $x$ is Low Then $y$ is Not Low, If $x$ is Low Then $z$ is Low (prop. 2). Rule 3 does not fit into ordinary defuzzifier settings but it easily does here.

Given $x, \operatorname{Low}(y)$ is fixed by rule 2 . So, in rule $1,1-\operatorname{Low}(y)$ is an upper bound for $\operatorname{Low}(x)$ : unless $\operatorname{Medium}(x)$ and $\operatorname{Low}(x)$ verify some restrictions - Low $(x) \leq 1-\operatorname{Medium}(x)$-, contradiction arises : either the rules or the concepts (MF's) used in them are wrong.

\section{FUNCTIONAL INTERPRETATION OF RULES.}

In the following, subsethood (prop. 1) is extended to rules "If $\boldsymbol{x}$ is $A$ Then $\boldsymbol{y}$ is $B$ " when modelling functions $f: U \subset \mathfrak{R}^{n} \rightarrow V \subset \Re$ with fuzzy antecedents and consequents over $U, V$. It provides interesting conclusions for applications in which fuzzy systems are used as function approximators (typically, fuzzy controllers).

Definition 9. A function $f$ is coherent with a rulebase if $f(x)$ is a coherent conclusion (Def. 8) for premise $x, \forall x \in U$.

The extension principle [13] is the starting point in the exposition: a function $f: U \rightarrow V$ can be extended defining the image of a fuzzy set $A \subset U$ by: $\quad \mu_{f(A)}(y)=\sup _{f(x)=y} \mu_{A}(x)$

Conversely, the inverse image of a set $B \in \Pi(V)$ is given by:

$$
\mu_{f^{-1}(B)}(x)=\mu_{B}(f(x))
$$

For bijective $f$, (4) transforms into: $\mu_{f(A)}(f(x))=\mu_{A}(x)$

It can be shown that the following properties are satisfied:

$$
\begin{aligned}
& f\left(A \cup A^{\prime}\right)=f(A) \cup f\left(A^{\prime}\right) \\
& f\left(A \cap A^{\prime}\right) \subseteq f(A) \cap f\left(A^{\prime}\right) \\
& f^{-1}\left(B \cup B^{\prime}\right)=f^{-1}(B) \cup f^{-1}\left(B^{\prime}\right) \\
& f^{-1}\left(B \cap B^{\prime}\right)=f^{-1}(B) \cap f^{-1}\left(B^{\prime}\right) \\
& A \subseteq A^{*} \Rightarrow f(\mathrm{~A}) \subseteq f\left(A^{*}\right) \\
& B \subseteq B^{*} \Rightarrow f^{-1}(B) \subseteq f^{-1}\left(B^{*}\right) \\
& A \subseteq f^{-1}(f(A)) \\
& B=f\left(f^{-1}(B)\right)
\end{aligned}
$$

From (4), $\mu_{M}(x) \leq \mu_{f(M)}(f(x))$

and (6) gives $\mu_{f(M)}(f(x))=\mu_{M}(x)$, closely resembling (3).

Lemma 1. Given $f: U \rightarrow V$ and a fuzzy set $N \subset V, f$ is coherent with the rule " $x$ is $M \Leftrightarrow y$ is $N$ " if and only if $M=f^{-1}(N)$.

Proof. If $M=f^{l}(N)$ then : $\mu_{f^{-1}(N)}(x)=\mu_{N}(f(x))$, hence $y=f(x)$ satisfies (3), $\mu_{M}(x)=\mu_{N}(y)$ (Suff.).

If $f$ is coherent with the rule, $f(x)$ satisfies eq. (3) $\mu_{M}(x)=\mu_{N}(f(x))$ for all $x$, so it defines the set $M$ given $N$, as in (5), hence $M \equiv f^{-1}(N)$ (Necess.).

Corollary. If $f$ is coherent with the referred IIF rule, then $f(M)=N$. Proved from (12). The reciprocal is not true as (11) does not hold, in general, if $\subseteq$ is replaced with an = sign. Thus, lemma 1 is not valid if stated with $f(M)=N$ instead of $M=f^{-1}(N)$.

Lemma 2. Given a function $f: U \rightarrow V$ and a fuzzy set $N \subset V$, the function is coherent with the rule "IF $x$ is $M$ THEN $y$ is $N$ " if and 
Fuzzy Systems Evaluation. The Inference Error Approach.

\begin{tabular}{|c|c|c|c|}
\hline $\mathrm{A}$ & $\mathrm{B}$ & $\varepsilon_{\mathrm{IIF}}$ & $\varepsilon_{\mathrm{IF}}$ \\
\hline 0 & 0 & 0 & 0 \\
\hline 0 & 1 & 1 & 0 \\
\hline 1 & 0 & 1 & 1 \\
\hline 1 & 1 & 0 & 0 \\
\hline
\end{tabular}

Table 1. Inference error definitions (binary case).

only if $M \subseteq f^{-1}(N)$ (or equivalently $f(M) \subseteq \mathrm{N}$ ).

Proof. If $M \subseteq f^{-1}(N)$ then $\mu_{M}(x) \leq \mu_{f^{-1}(N)}(x)=\mu_{N}(f(x))$ so,

$f(x)$ satisfies (3) (Suff.).

If $f$ is coherent with the rule, $\mu_{M}(x) \leq \mu_{N}(f(x))$, i.e. less or equal to that given by (5), so $\mathrm{M}$ is a subset of $f^{-1}(N)$. (Necessity)

Observing the difference between (11) and (12), and the difference between $\cap$ and $\cup$ in (7), the following definition names a group of sets that do not have those differences.

Definition 10. Given $f: U \rightarrow V$, if $M \subset U$ verifies $f^{-1}(f(M))=M$ then it will be named as proper antecedent for function $f$. Equivalently, $M$ is a proper antecedent if it exists a fuzzy set $N$ in $V$ such that $M=f^{-1}(N)$. Note that if $f$ is bijective, all antecedents are proper.

By application of lemma 1, only if $M$ is a proper antecedent then the rule "IF and only IF $x$ is $M$ Then $y$ is $f(M)$ " is coherent with $f$. The analogous IF rule is also coherent (Lemma 2). Proper antecedents and IIF rules present a function as a bijective one: the consequent is the image of the antecedent and vice-versa, i.e. a "one to one mapping" between sets (not between set elements).

Interesting properties derived from (4)-...-(13) hold for proper antecedents. They help to identify and set them up in practice:

- For any $N \in V, f^{-1}(N)$ is a proper antecedent (12).

- If and only if $M$ is a proper antecedent, it satisfies

$$
\begin{aligned}
& \text { - } f\left(x_{1}\right)=f\left(x_{2}\right) \Rightarrow \mu_{M}\left(x_{1}\right)=\mu_{M}\left(x_{2}\right) \\
& \text { - Even with } f \text { not bijective, } \mu_{f(M)}(f(x))=\mu_{M}(x)
\end{aligned}
$$

Proposition 4. Union and intersection of proper antecedents is also a proper antecedent. Proof omitted for brevity.

\section{A. Intuitive interpretation of the lemmas.}

The intuitive meaning of the inverse function of a set $N$ may be such as "all the input conditions producing an output in $N$ ". So, the lemmas agree with intuition: "An $I F$ rule has in its antecedent some of the inputs in which its consequent is produced" , "An IIF rule has in its antecedent all of them".

This interpretation and (11) can be cast together: the outputs for input M (i.e., $f(M)$ ) are a subset of $N$. That implies either that $M$ could be "extended" up to including all situations in $N$ (i.e. being proper) or that $N$ could be "reduced" to include only the actions in $f(M)$. The rules in which $N$ cannot be "reduced" will be now defined:

Definition 11. Rules in which $f($ Antecedent $)=$ Consequent are called proper rules with respect to function $f$.

All coherent IIF rules are proper but some $I F$ rules are not.

Example (nonfuzzy). Being $f(x)=x^{2}, x \in[-5,5], " ~ x$ is $\{2,3\} \Rightarrow y$ is $\{4,9,16\}$ " is coherent with $f$. Lemma 2 holds as $\{2,3\} \subseteq\{2,-2$, $3,-3,4,-4\}=f^{-1}(\{4,9,16\})$, but the rule is not proper. The proper one is " $x$ is $\{2,3\} \Rightarrow y$ is $\{4,9\}$ ". The proper antecedent of $\{4,9\}$ is $\{-2,2,-3,3\}$ : "IIF $x$ is $\{-2,2,-3,3\}$ Then $y$ is $\{4,9\}$ ".

Given a function and an antecedent $M$, proper rules have the
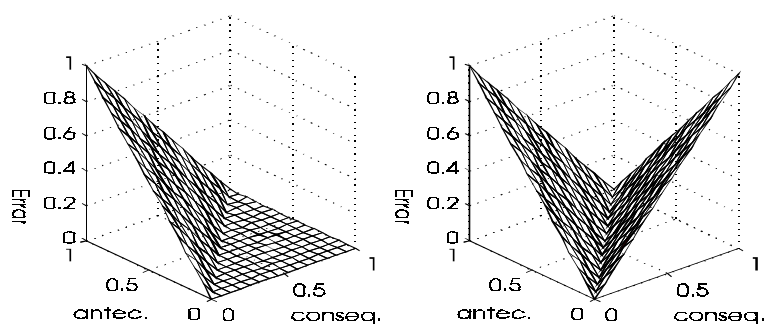

Fig. 1. Fuzzy inference error .

"minimum" (most precise) consequent $N$. All supersets of $N$ form coherent IF rules. Conversely, for a given consequent $N$, proper antecedents refer to a "maximum" antecedent $M$. All subsets of $M$ form coherent IF rules with $N$.

Proposition 5. If rules " $\mathrm{A} \Rightarrow \mathrm{B}$ ", " $\mathrm{A} * \mathrm{~B}$ *" are coherent with a function $f$, then " $\mathrm{A} \cap \mathrm{A} * \Rightarrow \mathrm{B} \cap \mathrm{B}^{*}$ " and " $\mathrm{A} \cup \mathrm{A} * \Rightarrow \mathrm{B} \cup \mathrm{B}$ *" are also coherent. The proposition also holds for IIF rules. Proof: If $A \subseteq f^{-1}(B), A^{*} \subseteq f^{-1}\left(B^{*}\right)$, then $A \cap A^{*} \subseteq f^{-1}(B) \cap f^{-1}\left(B^{*}\right)$ and $A \cup A^{*} \subseteq f^{-1}(B) \cup f^{-1}\left(B^{*}\right)$, and then apply (8).

Proposition 6. Proper rules satisfy MF peak equality: $\max _{u \in U} \mu_{A}(u)=\max _{y \in Y} \mu_{C}(y)$. Proof easily derived from (4).

Proposition 5 enables rule combination guaranteeing coherence but, in general, proper IF rules only remain proper if combined with the union operator (7). Properness precisely defines intuitively "useful", "well-behaved" rules. If improperness is present in some rules, it may lead to rulebase incompleteness.

\section{INFERENCE ERROR}

Up to this point, inference over coherent rulebases and transformations that keep coherence have been established. This section presents approximate inference with inconsistent rulebases, keeping previous results and intuition. The approach is based on a cost index minimisation. The index is a conceptual distance from a candidate conclusion to the conclusion set of each rule. This distance will assess how many rules are in contradiction if a conclusion is assumed.

A function $\varepsilon:[0,1] \times[0,1] \rightarrow \mathfrak{R}^{+}$-rule inference error $(\mathbf{I E})$ - is defined, such that $\forall x, y \in[0,1] \varepsilon(x, y) \geq 0$ and $\varepsilon(x, y)=0 \Leftrightarrow x$ is a solution of the rule equation. In the binary case, IE is a function $\{0,1\} \times\{0,1\} \rightarrow \Re^{+}$. When a conclusion is inconsistent it is defined as 1 to denote that one rule is contradicted (Table 1).

Definition 12. Once the inference error for each rule has been defined, the cost index formed by adding all the individual error functions will be called global inference error of the rulebase.

The global IE is equal to the number of contradicted rules for a particular combination of premises and conclusions. IE will be null only at the solutions of the equations. Thus, inference consists on finding the zeroes of the global IE.

Definition 13. The inference error functions $\varepsilon:[0,1] \times[0,1] \rightarrow[0,1]$ for fuzzy IIF and IF rules are (see Fig. 1) :

$$
\begin{aligned}
& \varepsilon_{I I F}(A, B)=|B-A|^{p} \\
& \varepsilon_{I F}(A, B)=\left\{\begin{array}{cc}
0 & A \leq B \\
(A-B)^{p} & A>B
\end{array}\right.
\end{aligned}
$$

where $p>0$ is a design parameter with reference value $p=1$. Higher values relatively reduce small errors and lower do the opposite.

IE functions are a gradation of contradiction: a conclusion $B$ may be coherent with premise $A$ and a rule $(\varepsilon=0)$, it may be fully 


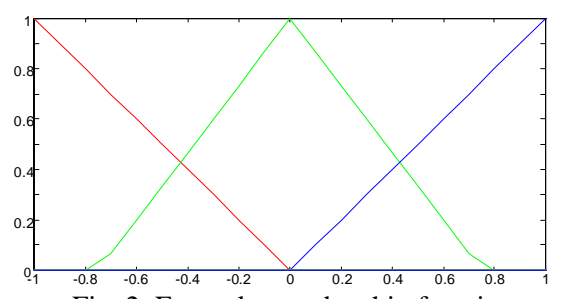

Fig. 2. Example membership functions.

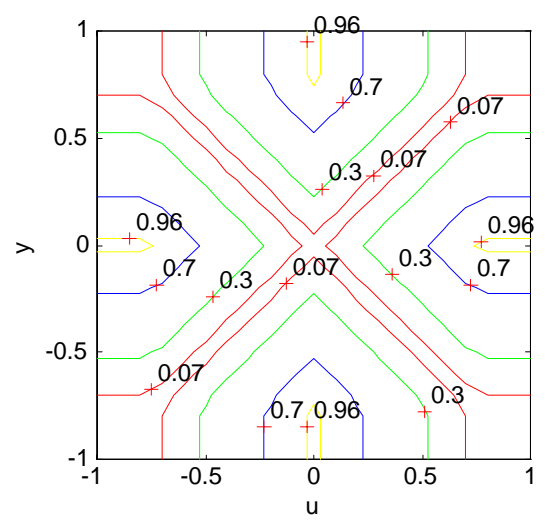

Fig. 3. Fuzzy IE curves for $\boldsymbol{u}$ is Zero $\Leftrightarrow \boldsymbol{y}$ is Zero

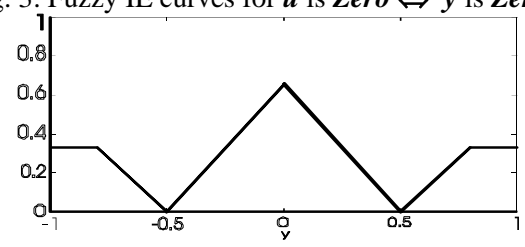

Fig. 4. Inference error for $u=-0.5$.

\begin{tabular}{|ccccc|}
\hline $\mathbf{u}$ & $\mathbf{y}$ & Zero(u) & Zero(y) & $\boldsymbol{\varepsilon}$ \\
\hline $\mathbf{- 1}$ & -1 & 0 & 0 & 0 \\
\hline$-\mathbf{1}$ & 0 & 0 & 1 & 1 \\
\hline $\mathbf{- 1}$ & 1 & 0 & 0 & 0 \\
\hline $\mathbf{0}$ & -1 & 1 & 0 & 1 \\
\hline $\mathbf{0}$ & 0 & 1 & 1 & 0 \\
\hline
\end{tabular}

Table 2. Some error values for $\mathrm{u}$ is Zero $\Leftrightarrow \mathrm{y}$ is Zero

contradictory $(\varepsilon=1)$ or it may lie somewhere in between.

When combined with fuzzification, the following IE functions $\varepsilon: U \times V \rightarrow[0,1]$ are obtained $(a$ and $c$ superscripts stand for antecedent and consequent MF's, respectively):

$$
\begin{gathered}
\varepsilon_{I I F}(u, y)=\left|\mu^{a}(u)-\mu^{c}(y)\right|^{p} \\
\varepsilon_{I F}(u, y)= \begin{cases}0 & \mu^{a}(u) \leq \mu^{c}(y) \\
\left(\mu^{a}(u)-\mu^{c}(y)\right)^{p} & \mu^{a}(u)>\mu^{c}(y)\end{cases}
\end{gathered}
$$

Rules are thus substituted by an equivalent IE representation. A rulebase can be represented by aggregating the individual rule IE functions ( $n$ is the total number of premises and conclusions, $N$ the number of rules, and $U, V, W, \ldots$ the universes of discourse of all premises and conclusions):

$$
\begin{array}{r}
\varepsilon: U \times V \times W \times \ldots \rightarrow[0, \infty) \\
\varepsilon(x)=\sum_{i=1}^{N} \phi_{i}(x) \varepsilon_{i}(x)
\end{array}
$$

where $x=\left(x_{1}, x_{2}, \ldots, x_{n}\right)$ is a vector consisting of all known premises and unknown conclusions, $\varepsilon_{i}$ are the individual IE functions and $\phi_{i}: U \times V \times W \times \ldots \rightarrow(0, \infty)$ are user-defined confidence functions that express the relative importance a rule may have (in practice, they are constant confidence levels).
Once defined the IE function $\varepsilon(x)$ for a rulebase, a redefinition of inference enables concluding from non-coherent rulebases.

Definition 14. Given a rulebase, the ideal conclusion for some premises is the set of values that minimise $\varepsilon(x)$.

Minimising $\varepsilon$ is equivalent to minimising the number of rules in contradiction weighted by their confidence level: ideal inference finds a "central" conclusion given each rule's conclusion set, calculated on the basis of the presented conceptual distance (IE contradiction measure), not on geometric distance.

Remark. Although in a general case ideal inference may involve complex minimum search algorithms, piecewise linear MF's produce piecewise linear IE curves, so successful implementation of ideal inference is feasible with low computer requirements. If MF's are carefully arranged, other inferencedefuzzifying algorithms, as centroid ones, provide consistent conclusions (see section 5).

Definition 15. Given a rulebase $\boldsymbol{B}$ with IE function $\varepsilon: U \times \Re \rightarrow \Re$ and a function $f: U \rightarrow \Re$, being $U$ the domain of the premises of $\boldsymbol{B}$, the error at $(u f f(u))$ is named as coherence mismatch index between $\mathrm{R}$ and $f$ at $u \in U$. The index denotes how many rules are in contradiction if the fuzzy system tries to model $f$ or conversely if $f$ tries to model ideal inference on $\boldsymbol{B}$ (section 5).

Example. Let us consider the triangular MF's (for input and output) labelled Negative, Zero, Positive, depicted in Fig. 2. Considering the rule "IIF $u$ is Zero Then $y$ is Zero", its IE is given by $\varepsilon(u, y)=\mid \operatorname{Zero}(u)$-Zero $(y) \mid$. Some values are presented in table 2 . For example, for premise $u=-1$, the conclusion set is $\mathrm{y}=\{-1,1\}$.

IE for rule 1 is plotted in the contour map in Fig. 3. Table 2 is a particular case. For premise $u=-0.5$, the error curve as a function of $y$ (Fig. 4) is given by: $\varepsilon(y)=|0.33-Z e r o(y)|$, i.e., a vertical $(u$ constant) cut on the represented surface. When $u$ is "Not Zero" it may be either positive or negative, so incompleteness arises.

As a further example, the following rulebase can be analysed: ["IIF $u$ is Positive Then $y$ is Positive", "IIF $u$ is Negative Then $y$ is Negative", "IF $u$ is Zero Then $y$ is Not Zero" $\}$. It is contradictory when $u$ is Zero : $\varepsilon(0,0)=1, \ldots, \varepsilon(0,-1)=1, \varepsilon(0,1)=1$. No contradiction arises if $u$ is not significantly "Zero". IE would have changed if different confidence levels had been introduced: if the third rule had a confidence level of 0.5 , the error at $(0,0)$ would have been 0.5 , hence the ideal inference would have concluded that for premise $u=0$ the "optimum" conclusion is $y=0$, because even contradicting rule 3 , other "more confident" ones say that it is the correct conclusion.

\section{VALIDATION OF FUZZY SYSTEMS.}

A fuzzy control system can be considered as a black-box with a set of input and output physical variables that produces a function approximation. Internally, fuzzification, inference and defuzzification are carried out (see [2], [6], [10], etc.).

The IE coherence mismatch (def. 15) validates a rulebase approximating a function. It may be used in the opposite way: given a fuzzy rulebase, the function encoded in it is recovered by finding the $f$ that makes IE as low as possible. If $f$ is restricted to be a classical technique (fuzzy relations, centre of mass, etc.), a validation method is thus defined: If $f(x)$ is the output of a fuzzification-inference-defuzzification paradigm over a coherent rulebase, its coherence mismatch is a quality measure of valitidy.

Thus, IE minimisation may not need to be actually 


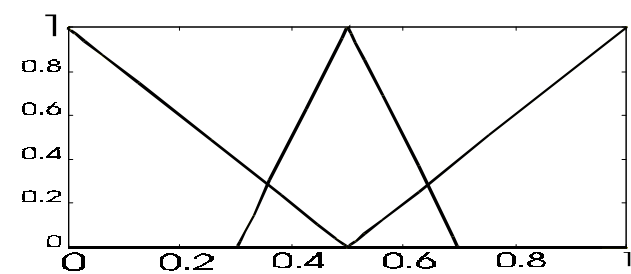

Fig. 5. Memberships for validation example.

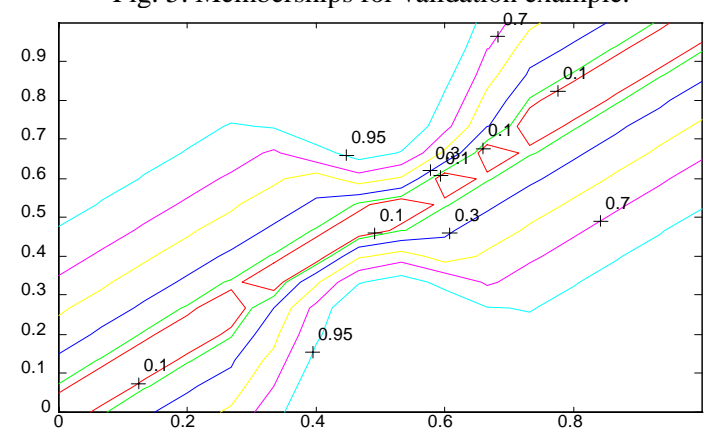

Fig. 6. Intuitive modelling of $y=x$ - IIF rules.

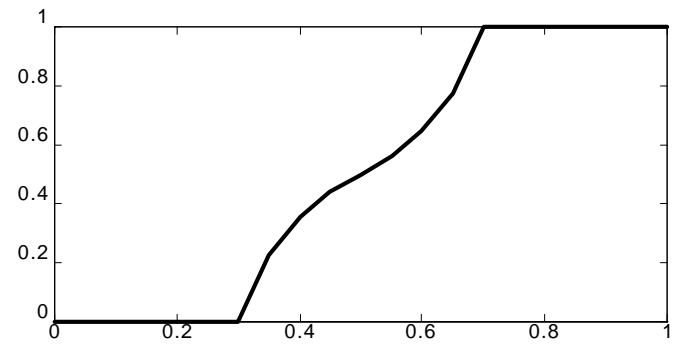

Fig. 7. Centroid defuzzifier output.

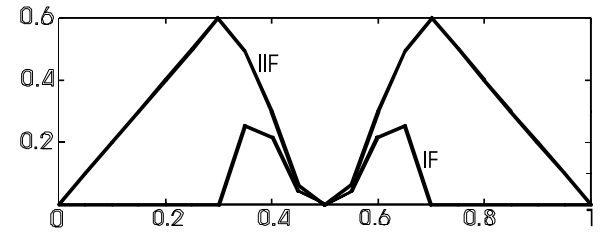

Fig. 8. IE of the centroid algorithm as a function of $x$

implemented in a particular system. If the conclusions of the actual algorithm (for example, centroid defuzzification) have a low inference error, its validity is asserted. Nevertheless, the validity depends on particular rules and MF arrangements. For example, with fat shape or non-convex sets (NotIntermediate = Small oR Big), centroid methods may provide invalid outputs. In that case MF rearrangement or changes in the defuzzifier algorithm are needed: The IE approach can be used to assess which one of various conventional inference algorithms, and in which circumstances, is best, based on algebraic and extension principle considerations, and not on mere heuristic reasoning.

Example. Given the MF's (Low, Medium, High) identical for input and output depicted in Fig. 5, the rulebase probably given by an expert modelling the identity function $y=x$ would be :

$\{x$ low $\Leftrightarrow y$ low,$x$ medium $\Leftrightarrow y$ medium,$x$ high $\Leftrightarrow y$ high $\}$

Representing the rules by $\operatorname{Low}(x)=\operatorname{Low}(y), \ldots$, and using the IE functions (16), the global IE is shown in Fig. 6. $y=x$ is the only coherent function, as intuitively expected. However, with the proposed input MF's, if a center of mass-like defuzzification :

$$
\text { out }=\frac{\sum \mu_{i}(x) \bar{y}_{i} A_{i}}{\sum \mu_{i}(x) A_{i}}
$$

is used, no values of $\bar{y}_{i}, A_{i}$ can be found to produce $y=x$. For
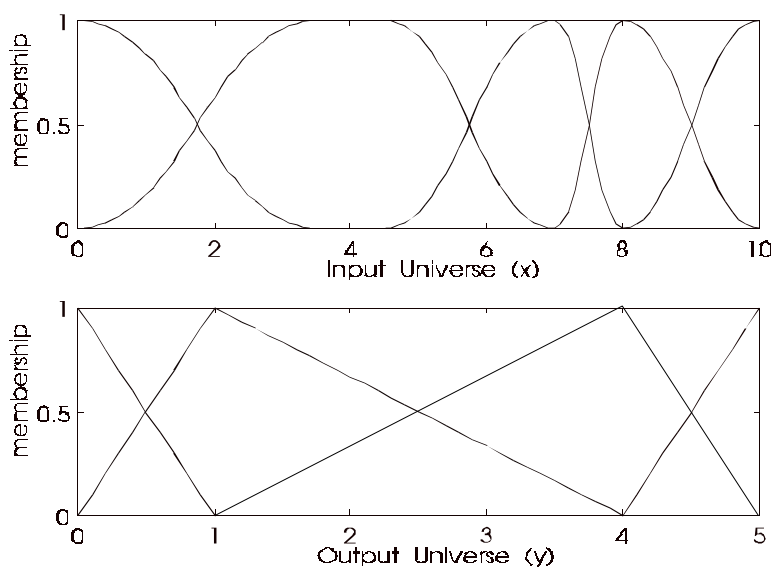

Fig. 9. Memberships for coherent CDG defuzzification.

example, for output centroids $(0,0.5,1)$ and $A_{i}=1$ the output in Fig. 7 is produced.

Superimposing Fig. 7 and Fig. 6 the defuzzifier can be validated with respect to consistency. The IE obtained for the centroid algorithm (as a function of $x$ ) is shown in Fig. 8, both for the IIF and IF rulebase versions. Around 0.3-0.4 and 0.6-0.7 the output is most contradictory: 0.25 (IF rules), 0.6 (IIF rules). As IIF rules are more 'specific', the error is bigger.

Hence, contradiction arises from the defuzzifying method and not from the rulebase. Note that an "ideal" algorithm should reproduce the identity function as it is the only coherent one.

The procedure can be applied to validate any fuzzy system. A widely used one will be considered to illustrate the approach.

Definition 16. A set of MF's $\mu_{k}(u) k=1, \ldots, n$ defined over an interval $[a, b] \subset \mathfrak{R}$ is an add-1 normal fuzzy partition if there exists a set of points $\left\{u_{j}\right\}$ such that $u_{j}<u_{j+1} \quad j=1, \ldots, n ; u_{1}=a$, $u_{n}=b$, the MF's satisfying the following conditions:

- $\mu_{k}(u)=0 \quad \forall u \notin\left[u_{k-1}, u_{k+1}\right], 1<k<n ; \mu_{k}\left(u_{k}\right)=1, k=1, \ldots, n$

- $\mu_{1}(u), \mu_{n}(u)=0$ outside $\left[a, u_{2}\right]$ and $\left[u_{n-1}, b\right]$, respectively

-in the interval $\left[u_{k}, u_{k+1}\right] \mu_{k}(u)+\mu_{k+1}(u)=1 \quad k=1, \ldots, n-1$

Note that these sets are fuzzy mutually exclusive (Def. 3 ).

If $\mu_{k}(u) \quad 1<k<n$ are triangular fuzzy sets, the partition is named triangular add-1 normal partition.

Lemma 3. If a fuzzy system has the structure:

1. $n$ input MF's $\mu_{k}^{i}(u)$ for fuzzy sets $\mathrm{I}_{\mathrm{k}}$ defined in such a way that they conform and add-1 normal fuzzy partition over a 1dimensional input universe [a,b] (Fig. 9),

2. $m$ output MF's for fuzzy sets $\mathrm{O}_{\mathrm{k}}$ defining a triangular add-1 normal partition (Fig. 9),

3. a rulebase formed by $n$ rules: $r_{p q}$ : "IF $u$ is $\mathrm{I}_{\mathrm{p}}$ Then $y$ is $\mathrm{O}_{\mathrm{q}}$ " ordered in the form $\left\{r_{1 \mathrm{c}(1)}, r_{2 \mathrm{c}(2)}, \ldots, r_{n \mathrm{c}(n)}\right\}$

so that for any two consecutive rules, their consequents verify $|\mathrm{c}(i)-\mathrm{c}(i+1)| \leq 1$ (output MF's are either adjacent or identical), then the following results hold :

a) The rulebase is coherent.

b) The centroid defuzzification method (equivalently, the one-dimensional lookup-table linear interpolation):

$$
y^{*}(u)=\left(\sum_{i=1}^{n} \mu_{i}(u) y_{c(i)}\right) \cdot\left(\sum_{i=1}^{n} \mu_{i}(u)\right)^{-1}
$$

is coherent (performs ideal inference) for $u \in\left[u_{1}, u_{n}\right]$.

c) If $\forall \mathrm{i}|\mathrm{c}(i)-\mathrm{c}(i+1)|=1$, the rulebase is complete so this defuzzifier produces the only possible coherent function. 


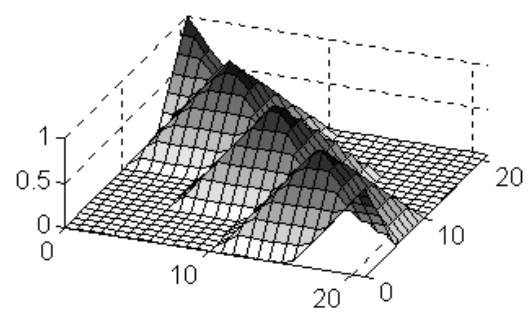

Fig. 10. 2-D antecedents for PD controller.

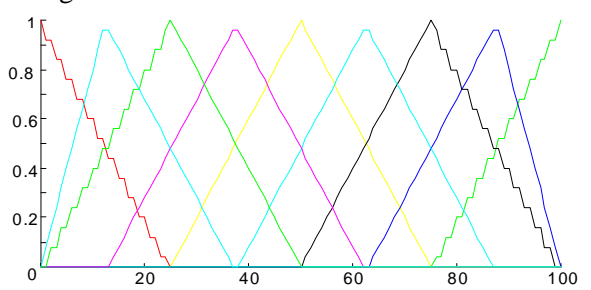

Fig. 11. Consequents for linear $f(e, \dot{e})$.

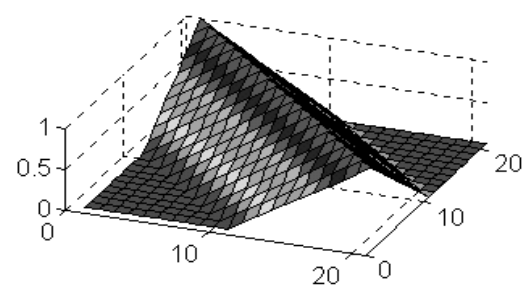

Fig. 12. Proper antecedent of a consequent in Fig. 11.

Corollary. If $|\mathrm{c}(i)-\mathrm{c}(i+1)|>1$ the rulebase is contradictory.

Proof: The denominator term in (19) equals 1 for all $u$ in the domain. If only one rule (say rule $j$ ) fires with strength 1 , the input is obviously $u_{j}$, and the defuzzifier output is $y^{*}=y_{c(j)}$. This value is coherent with rule $j$ as: $\mu_{c(j)}\left(y_{c(j)}\right)=1 \geq \mu_{j}\left(u_{j}\right)=1$, as well as with the rest of the rules:

$$
\mu_{c(k)}\left(y_{c(j)}\right)=0 \geq \mu_{k}\left(u_{j}\right)=0 \quad \forall k \neq j .
$$

If two consecutive rules $j$ and $j+1$ are fired so $u \in\left(u_{j}, u_{j+1}\right)$, ideal inference produces the following conclusion sets $C_{k}(u)$ :

- "anything" for non-fired rules : $C_{k}(u)=[\mathrm{a}, \mathrm{b}] \quad k \neq j, j+1$

- the interval in which output membership is greater or equal to the input one, for rules $j$ and $j+1$, according to equation (3) :

$$
\begin{aligned}
& C_{j}(u)=\left[\mu_{j}(u) y_{c(j)}+\left(1-\mu_{j}(u)\right) y_{c(j)-1},\right. \\
& \left.\mu_{j}(u) y_{c(j)}+\left(1-\mu_{j}(u)\right) y_{c(j)+1}\right] \\
& C_{j+1}(u)=\left[\mu_{j+1}(u) y_{c(j+1)}+\left(1-\mu_{j+1}(u)\right) y_{c(j+1)-1},\right. \\
& \left.\mu_{j+1}(u) y_{c(j+1)}+\left(1-\mu_{j+1}(u)\right) y_{c(j+1)+1}\right]
\end{aligned}
$$

Taking into account that $1-\mu_{j}(u)=\mu_{j+1}(u)$ and that if the output functions are consecutive, as stated in assumption 3 of the lemma, then either $c(j)-1=c(j+1)$ or $c(j)+1=c(j+1)$ is true, i.e. either the left or the right endpoint of $C_{j}(u)$ is an endpoint of $C_{j+1}(u)$ and that point is exactly the interpolative defuzzifier output $y^{*}$. Note that, as the intersection of the two conclusion sets has only one element in it, the rulebase is coherent and complete. If $\mathrm{c}(j)$ or $\mathrm{c}(j+l)$ are 1 or $m$, the treatment is similar. If consequents are identical $c(j)=c(j+1)$, (19) yields $y^{*}=y_{c(j)}$, i.e. the peak membership point of the conclusion sets, hence a coherent solution (but not the only one).

Lemma 3, stated for single input functions, cannot be fully extended to multi-input fuzzy systems with antecedents conformed by using the AND connective among add- 1 partitions for each input. These antecedents conform a partition of the input space in fuzzy "tiles" (hypercubes in general) that may be not the most suitable to model arbitrary $n$-dimensional surfaces.

Nevertheless, interesting properties can also be derived for them. To show the additional difficulties, extension of this lemma to 2-input functions $f: U \times V \subset \Re^{2} \rightarrow \Re, y=f(u, v)$ will be considered.

The MF arrangement to be analysed will be that in which two 1-dimensional add-1 normal fuzzy partitions with $m$ and $k$ fuzzy sets, respectively, are defined over two intervals $U$ and $V$ that act as input universes. The 2-D domain is partitioned into $m \times k$ fuzzy sets by using the AND (minimum) connective. MF's will be assumed continuous, as well as the function $f$ to be modelled.

Coherent consequents must be obtained from these antecedents via the extension principle, so $m \times k$ rules are built in the form:

$$
r_{s p q} \text { : "IF }\left(u \text { is } \mathrm{I}_{\mathrm{s}}\right) \text { and }\left(v \text { is } \mathrm{J}_{\mathrm{p}}\right) \text { Then } y \text { is } \mathrm{O}_{\mathrm{q}} \text { " }
$$

where $\mathrm{O}_{\mathrm{q}}$ is the image of the antecedent with respect to $f$. The rules are arranged in the rule array: $\left\{r_{11 \mathrm{cc}(1,1)}, r_{12 c(1,2)}, \ldots, r_{m k c(m, k)}\right\}$

For linear $f$ and triangular input MF's, overlapping triangular fuzzy consequents form coherent proper rules (see example A in this section). This is important from the practical point of view: linear controllers can be modelled by a coherent fuzzy system with proper rules with triangular sets. Nonlinear functions may be also modelled by this kind of MF's and coherent rulebases, but rules lose their properness if triangular output consequents are kept (they must be supersets of the non-triangular proper ones).

With the described input MF's, equal membership zones in the $U \times V$ input space are rectangular (maybe with "thick" edges corresponding to equal membership intervals in the 1-D functions). These rectangles surround at least one "center" point with membership 1, contained in its interior, corresponding to the peak membership points of the 1-D MF's. Those centers define a kind of "fuzzy grid". Any point in the input space has nonzero membership in at most 4 sets : no more than 4 rules can be fired simultaneously.

Notwithstanding, the use of these simple antecedents intuitively implies the assumption that function $f$ has local behaviour around the peak points similar to the referred "tiles" (i.e., that those tiles are defined by fuzzy sets that group qualitatively similar function outputs). If $f$ does not have that response, maybe the antecedent arrangement here analysed is not the most efficient one. Not even linear functions have this property (see example A). In fact the possibility of incompleteness arises due to improperness in antecedents. Note that this incompleteness is neither counter-intuitive nor so detrimental : in the general case, there are various ambiguous alternatives to carry out 2-D interpolation between 4 points.

Proposition 7. In the presented arrangement it can be shown that it does not exist a continuous function $f$ for which all the antecedents are proper. The proof is omitted for brevity.

The following examples illustrate improperness, even with linear functions, and asses the incompleteness level.

\section{A. Application examples: P, PD (PI) fuzzy controllers.}

The MF organisation and defuzzifiers just analysed are widely used to model nonlinearities in P, PD and PI fuzzy controllers.

Proportional controllers $(u=\mathrm{k} \cdot e)$ obtained from add-1 normal fuzzy partitions at the input variable are coherent, and centroid defuzzification can be used (lemma 3 ) in fuzzy models of a linear or piecewise linear $\mathrm{P}$ controller with triangular input MF's. 


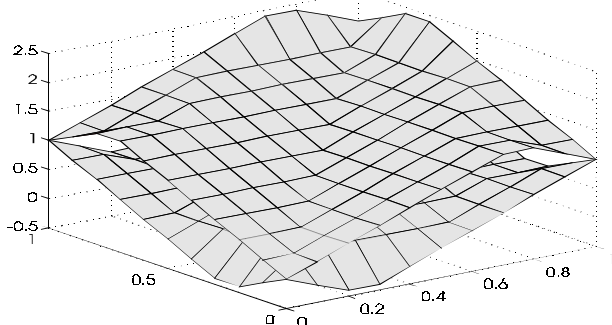

Fig. 13. Incompleteness due to improper antecedents.

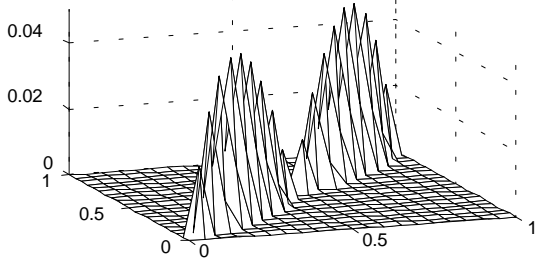

Fig. 14. Inference error for 2-D centroid defuzzifier

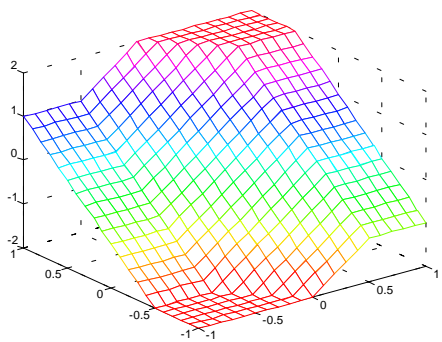

Fig. 15. Characteristic surface of a nonlinear controller.
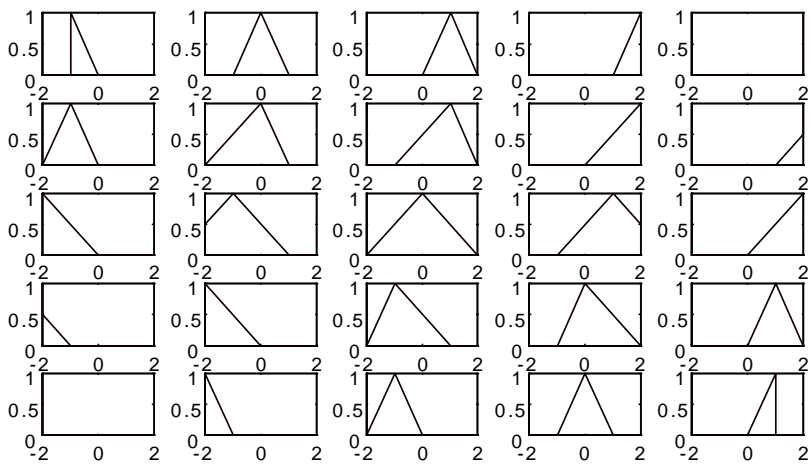

Fig. 16. Consequents for proper rules.

In 2-variable PD-like fuzzy controllers $u=f(e, \dot{e})$ rule antecedents are similar to the ones shown in Fig. 10 (from two triangular input partitions). They produce images, if a linear PD controller is modelled, as shown in Fig. 11. Notwithstanding, proper antecedents (inverse image) for those consequents have the shape described in Fig. 12. The antecedents in Fig. 10 are subsets of these ones. This fact gives rise to incompleteness in some zones of the input space. For example, with $y=u+v$, $u \in[0,1], v \in[0,1]$ with two linear fuzzy sets on each input universe, all possible coherent interpolations are the functions between the two surfaces shown in Fig. 13.

The centroid inference-defuzzification algorithm given by:

$$
\begin{aligned}
& \eta_{j}=\max _{\left\{a, b \mid \exists r_{a b j}\right\}}\left(\min \left(\mu_{a}(u), \mu_{b}(v)\right)\right) \\
& y^{*}(u, v)=\sum_{j=1}^{m} \eta_{j} y_{j} / \sum_{j=1}^{m} \eta_{j}
\end{aligned}
$$

is not fully coherent with the rule base, but it is coherent in almost all the domain, and its maximum inference error is 0.05 at the most incoherent points (for $y=a+b$ ), as shown in Fig. 14. Hence, it

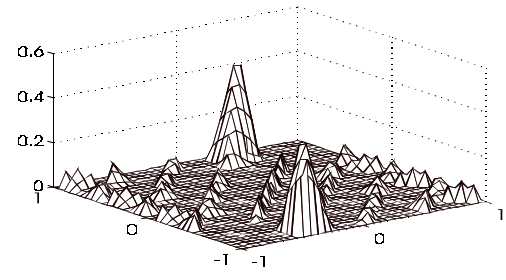

(A) weight $=1$

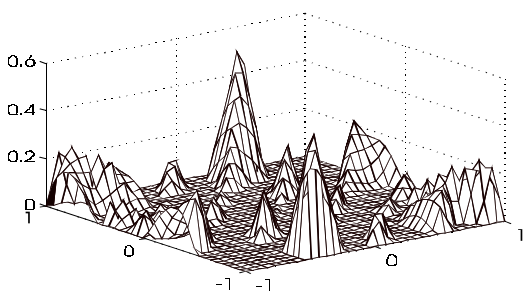

(B) weight $=$ area

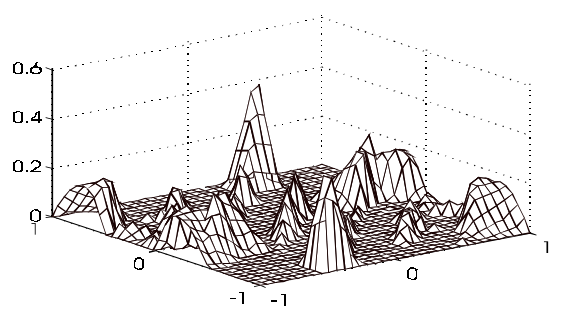

(C) weight $=1 /$ area

Fig. 17. Inf. error for CDG defuzzifier.

can be used in practice with linear 2-D functions to yield nearly ideal inference results.

\section{B. Nonlinear fuzzy controllers.}

One of the main reasons to use fuzzy systems in control is their ability to model nonlinearities. In the same way that linear or piecewise linear P-like controllers have been analysed, general static nonlinear controllers $u=f(e)$ can be modelled by using nonlinear input MF's (for example, polynomial-based ones, as in Fig. 9). In most practical cases these nonlinear controllers implement monotonic functions. The application of the extension principle to those functions is simple and intuitive.

As an example of a two-variable nonlinear design, a PD controller with control action and derivative saturation will be designed to be implemented on a fuzzy system. The controller must achieve the control surface plot shown in Fig. 15.

Two add-1 normal partitions on error $(e)$ and error derivative $(\dot{e})$ inputs with triangular MF's will be defined. Each partition will have 5 fuzzy sets \{negative big, negative small, zero, positive small, positive big $\}$, with peaks at $-1,-0.5,0,0.5,1$ respectively. They define 25 fuzzy 2-D antecedents. The consequents, calculated by the extension principle, are represented in Fig. 16.

For $\mathrm{e}=\mathrm{PB}$ AND $\mathrm{de}=\mathrm{PB}$ the consequent is a singleton $(+2)$, as well as for $\mathrm{e}=\mathrm{NB}, \mathrm{de}=\mathrm{NB}(-2)$, because all the zone covered by the antecedent attains a constant value. An algorithm that does not produce exactly that value would contradict that rule. Note also that piecewise linear proper consequents are produced due to the selection of the antecedents and the particular geometry of the control surface. That would not be the case in a general situation.

Inference error of a centroid algorithm that uses the peak values as centroids, weighted by equal areas, produces an IE plot 
shown in Fig. 17-(A). If the weighting would have been proportional to areas, and inverse proportional to them, the inference error would have been the one shown in Fig. 17-(B) and Fig. 17-(C), respectively. Hence, in this case, the most valid defuzzifier is the one that does not take into account area weighting, as in the one-dimensional case.

Note that formal validation has been analysed for 1-D and 2-D controllers, in a very restricted set of MF design possibilities. The more the required properties are not fulfilled the more intuitive "problems" (now clearly formulated) pose the referred defuzzification methods. That is the reason why most of the practical implementations of fuzzy controllers have the MF arrangement as in lemma 3 or in the 2-D analysis.

\section{A. Rulebase design tips for valid centroid defuzzification.}

In order to keep the centroid defuzzification coherent, the following design tips for function approximation can be stated :

1. Rules have to be assumed of "IF" type.

2. Antecedents should have MF's conforming add-1 partitions.

3. Antecedent and consequent should be normal sets (peak 1).

4. Input and output MF's should verify extension principle (if the function to be modelled is explicitly known).

5. In fuzzy 1-D lookup tables (piecewise linear interpolation) consequents should be triangular add-1 normal partitions.

6. For 2-D functions,

(a)- if sufficient knowledge about the function geometry is available, proper antecedents as well as triangular add-1 consequents should be used to get perfect coherence.

(b)- Assuming linear objective functions, the use of improper antecedents obtained by AND operation over two triangular add-1 partitions with overlapping triangular consequents (Fig. 10) will result in satisfactory operation from the IE point of view. For arbitrary non-linear functions, centroid algorithms may be highly incoherent (as shown in the example with IE over 0.4) -this is an "inference error" version of the fact that these interpolations cannot model arbitrary functions in middle points- (If improper antecedents are used even ideal inference is not complete).

7. Centroids should be set equal to the values having peak output membership, in both 1-D and in 2-D arrangements. The examples here analysed suggest that the "area" consideration in the defuzzification formula does not improve the results.

8. Rules with neighbouring antecedent must have neighbouring consequents for the rulebase to be coherent.

If the MF arrangement is not similar to the ones here exposed centroid defuzzification may be a not valid inference algorithm. The worse situations occurs with nonconvex consequents when taking its center of mass or mean of maxima as centroid: even totally contradictory results $(\varepsilon=1)$ may be produced.

\section{CONCLUSIONS}

An equation-based representation of fuzzy logic rulebases has been presented taking into account the extension principle, the fuzzy subsethood formulas and the Lukasiewicz implication.

By this representation, rules may be understood as examples of a function to be modelled obtained by applying that function to some fuzzy sets. The concepts of proper rules and proper antecedents formalise intuitive notions such as equality of membership peaks and differences between IF and IIF rules.
By defining an error function related to the rule equations, ideal inference is defined in terms of minimisation of the global rulebase contradiction. Confidence levels stand as weights on the error function. Rulebase and defuzzifier validity can be formally checked. As one of the most prominent applications of fuzzy systems is fuzzy control, their use as function approximators has been discussed and the validation of common fuzzy inferencedefuzzification strategies for static nonlinearities $y=f(u)$ or $y=f(u$, v) for P and PD-like fuzzy controllers has been carried out. As expected, they are similar to ideal inference if consequents are triangular add- 1 partitions. They produce results with very low inference error in most of the commonly used cases.

The concepts here defined pave the way to further research on automatic knowledge acquisition supervision: detection of contradictions, rulebase simplification (elimination of redundant rules), multi-expert fusion and efficient learning.

\section{REFERENCES}

[1] P. Albertos, M. Martinez, J.L. Navarro, and F. Morant. "Fuzzy Controller Design : A methodology". Proc. of IEEE Conference on Control Applications, Vancouver 1993.

[2] P. Albertos, "Fuzzy Logic Modelling and Control". From Identification to Learning, pp. 479-513. Ed.: S. Bittanti. Springer-Verlag, 1996

[3] B. Cragun, and H. Steudel, "A decision-table-based processor for checking completeness and consistency in rule-based expert systems." Int. J. Man-Machine Studies, v. 26, no. 5. , pp 633-648, 1987.

[4] E.E. Kerre, "A comparative study of the behaviour of some popular fuzzy implication operators". From Fuzzy Logic for the management of uncertainty, Ed.: L.A. Zadeh and J. Kacprzyk, John Wiley \& Sons.1992

[5] F. Klawoon, J. Gebhardt and R. Kruse. "Fuzzy Control on the basis of equality relations with an example from idle speed control". IEEE Trans. Fuzzy Systems. Vol. 3, No. 3, pp. 336350, Aug. 1995.

[6] C.C. Lee, "Fuzzy Logic in Control Systems ; fuzzy logic controller part I/II" IEEE Trans. Systems, Man, Cybernetics. Vol 20, no. 2, pp.404-435, Mar./Apr. 1990.

[7] S. Lee, and R.M. O'Keefe, "Developing a Strategy for Expert System Verification and Validation" IEEE Trans. on Systems, Man and Cybernetics, vol. 24, no 4, pp 643-655, 1994.

[8] M. Suwa, A.. Scott, and E.H. Shortliffe, "An approach to verifying completeness and consistency in a rule-based expert system" AI Mag., pp 16-21. 1982.

[9] T. Whalen and B. Schott, "Presumption, prejudice and regularity in fuzzy material implication". Same source as [4].

[10] R.R. Yager, S. Ovchinnikov, R.M. Tong, and H.T. Nguyen, Fuzzy Sets and Applications. Selected papers by L.A. Zadeh. New York : John Wiley and Sons, 1987.

[11] W. Yu, and Z. Bien, "Design of Fuzzy Logic Controller with Inconsistent Rule Base". J. of Intelligent and Fuzzy Systems, Vol 2, pp. 147-159, 1994.

[12] L.A. Zadeh, "Fuzzy sets". Information and Control, vol 8, pp. 338-353, 1965.

[13] L.A. Zadeh, "Similarity relations and fuzzy orderings". Information Sciences, vol 3, pp.177-200, 1971. 